\title{
Report on the Ad-hoc Review of the IUGS Commission on Tectonics and Structural Geology (TecTask)
}

\author{
${ }^{1}$ ARC Chair - IUGS Vice-President \\ ${ }^{2}$ ARC Secretary - IUGS Councillor \\ ${ }^{3}$ ARC External Reviewer - Monash University \\ ${ }^{4}$ ARC External Reviewer - Pierre and Marie Curie University
}

https://doi.org/10.18814/epiiugs/2019/019029

\section{Rationale for the Review}

The IUGS Executive Committee (EC) is required by its Statutes and Bylaws to undertake a formal review of all commissions and other bodies funded by the Union every four years. The primary tasks of ad-hoc reviews are (i) to review the activities of those scientific bodies and (ii) provide accountability for the expenditures incurred by the Union. TecTask was established as a Task Group in 2004, and was subsequently upgraded to a Commission in 2009. It has never been subject to a formal review.

During the $71^{\text {st }}$ annual meeting of the IUGS EC, held in Paris in February, 2017, a decision was made to review TecTask within the next year. As a result, on March $27^{\text {th }}, 2017$, William Cavazza informed Rodolfo Carosi that an IUGS AdHoc Review of the TecTask Commission was to be scheduled for the fall of 2017. At that time Carosi was provided with the relevant documentation and information regarding the review process. Subsequently, in consultation with Carosi, it was confirmed on June $16^{\text {th }}, 2017$, that the Ad-hoc Review Committee (ARC) would convene in Turin on September 11-12, 2017. At this time Carosi was informed that the main goals of the review were to (i) analyze the past performance of the TecTask Commission, (ii) assess TecTask objectives for their alignment with the aims of the IUGS, and (iii) help identify ways to increase the impact and significance of the Commission, including the identification of concrete objectives for the near future. To carry out the review, an ARC was established (see composition above). The composition of the ARC was ratified by a vote of the EC on July $23^{\text {rd }} 2017$. On August $2^{\text {nd }}$ Carosi delivered the following TecTask materials to the ARC: copies of the annual reports for TecTask for the years covering 2013, 2014, 2015, 2016 and 2017, and a document describing TecTask and its overall objectives.

\section{Information to be Considered}

At the start of the review process in Turin, the ARC Chair outlined the Terms of Reference (TOR) of IUGS ARCs, presented background information for the review, reviewed the requirements for routine IUGS reviews of Commissions as per the Statutes and Bylaws, summarized the types of issues normally addressed during reviews, and outlined the overall process that would be followed during the TecTask review. Two of the main points highlighted were that 1) according to the IUGS Statutes and Bylaws, the normal lifespan of a commission is eight years, and 2) Commissions are to be goal oriented and are commonly tasked with putting in place standards or establishing baselines that can be employed by the broad Earth Science community.

The ARC Chair's opening comments included a reminder that the IUGS represents a group of $\sim 50$ scientific organizations, and that its activities are financially sponsored by Adhering Organizations. Convincing these Adhering Organizations to support the IUGS requires that the Union be able to point to direct benefits (added value) to the global Earth Science community. Examples of such direct benefits recognized as 'added value' include the development of IUGS common standards in - amongst others - stratigraphic nomenclature, geological maps, isotopic decay constants, and petrological nomenclature. From this perspective, the success of TecTask has to be judged on the basis of its concrete accomplishments relative to the financial support it is has received from the IUGS.

\section{Summary of the Interview with Dr. Rodolfo Carosi}

Carosi provided a presentation outlining the i) the nature of the field of Structural Geology and Tectonics, ii) the history and development of the TecTask Commission, iii) past achievements of TecTask, iv) its current structure and focus, and v) the proposed future outlook and goals of the Commission. The 
main goal of TecTask is to "encourage innovative research and continued education in Tectonics and Structural Geology, the growth of intellectual capital and hence the impact of our science on the wealth of the global society".

The current leadership of TecTask includes a 6-member Executive Committee and 14 officers. An additional 3 officers are to be appointed before the end of 2017. There is also an ongoing effort to identify a Junior Officer. IUGS financial support of TecTask has averaged \$5,000/year. Funding peaked at $\$ 7,000$ per year from 2009 to 2012 and has since dropped steadily to $\$ 3,000$ per year for the past two years. In total, the IUGS has invested more than $\$ 75,000$ in TecTask.

Past tasks and responsibilities of TecTask included:

- Outcropedia

- Henk Zwart Award

- TecTask Internet portal

- Outreach

- Organization and support of meetings and workshops

- Support of publication of special volumes

- Sponsorship of field trips

- Participation in conferences

- Short course development

- Geoheritage

However, many of these activities were moribund when Carosi took over the leadership of the commission in 2016. Under the new leadership an attempt at revitalizing the Commission is being made. Initiatives have included the development and launch of a new TecTask website, start-up of a Facebook site, and the start of an Outcrop of the Month photo competition (which will also be used to reinvigorate Outcropedia). Looking forward, the current TecTask leadership see 'Terminology and Standards', Outcropedia and Geoheritage as fundamental activities, the encouragement of African and Asian chapters, and the initiation of a monthly online seminar as key goals.

\section{Ad-hoc Review Committee Comments}

The following is a summary of the main concerns raised by the ARC.

\section{There has been a lack of comprehension on the part of TecTask regarding the rules governing and goals of an IUGS Commission}

It became clear over the course of the review that the TecTask Commission is operating not as an IUGS Commission but is instead playing the role of an international Structural Geology and Tectonics scientific association. Unlike most Earth Science fields of study, there is currently no international Structural Geology and Tectonics association. It is the opinion of the ARC members that there is a void in the structural geology community, and that such void has been somewhat improperly filled by TecTask.

A prime example of the improper association nature of TecTask is its 'membership'. Membership in TecTask is accomplished by registering through the TecTask webpage. Over 3200 people are currently registered as TecTask members. However, commissions are designed to consist of small groups tasked with focused goals. This is in contrast to TecTask membership which simply provides access to "software downloads" and "contact forms to submit ... questions". Another manifestation of the association nature of TecTask is it having put in place and awarding the Henk Zwart Award, an accolade aimed at recognizing scientists who have made an "outstanding contribution in elevating the science of structural geology, and have demonstrated excellence in structural geology". Awarding of such an annual honour should lie outside of the authority of a commission, which typically has an 8-year lifespan and hence cannot commit to ongoing responsibilities such as annual awards (although other commissions have established awards).

In the end, the ARC was of the opinion that much of the activity supported by TecTask would either have happened anyway (for example, the hosting of sessions at conferences, running field trips, publication of special volumes and books, and the organization of dedicated annual short-courses) or were just outside of the mandate of the commission.

\section{Some current TecTask goals are redundant or misguided}

Some of the stated goals of TecTask are redundant with other IUGS commissions and efforts, some are misguided, and some -while apparently justifiable- lack supporting data. For example, Geoheritage is listed as one of the main activities of TecTask and is featured on the TecTask webpage. The stated goal is to "raise awareness of geological heritage and geoconservation with (an) emphasis on structural geological and tectonic sites". However, this goal is redundant with the goals of the IUGS International Commission on Geoheritage (ICG).

In terms of misguided efforts, TecTask's outreach efforts seem particularly notable. Outreach involves communication with and education of the public. Typically outreach efforts are focused on providing information that is relevant to the location in which people live. TecTask has however defined outreach in such a way that includes, for example, publications in specialized scientific journals with no significance for the general public.

Finally, Outcropedia appears to be one of TecTask's justifiable efforts. The purpose of this initiative is to "make outcrops in unfamiliar places known and accessible to other geologists". At last count photographs of $\sim 700$ outcrops are available on the Outcropedia web page. The main problem is that there is no data to indicate whether this effort is achieving the desired outcome. Although there have been recent efforts to revitalize Outcropedia (such as the 'Outcrop of the Month' initiative), 
the web page is largely static (the same set of photographs have been available for years without any renewal, in part because there was no more memory space available), the photos are of low quality and often accompanied by sketchy captions, and there is no identified target audience (students or professionals? Geologists or the public?). Unfortunately, there is no data available to help focus improving Outcropedia. How frequently is the page accessed? Which photos are most often viewed? Who is viewing them? An additional question is whether Outcropedia as it stands is redundant with similar initiatives by universities worldwide, or if it may provide an added value to such initiatives (with for example some kind of interactive viewing or with improved links between pictures and established standards of nomenclature in structural geology and tectonics). It is, therefore, unclear whether Outcropedia is worth pursuing.

\section{TecTask leadership is currently too numerous and with- out specific goals}

The TecTask leadership group needs to be better aligned with the goals of the Commission. At present the leadership consists of a 6-member Executive Committee and 14 Officers. It is worth noting that despite the TecTask leadership having been notified of the Ad-hoc Review in March, only Dr. Carosi (Chair) made arrangements to attend and participate in the review. This lack of commitment on behalf of the current leadership is to some extent symptomatic of the problems with the TecTask Commission. At present only the Chair (Carosi), ViceChair (Mamtani) and Treasurer (Gomez-Rivas) are officially recognized by the IUGS Council. There is, therefore, an opportunity to reduce the size of the leadership group, and to co-opt a limited number of new officers tasked with meeting the specific new goals of the TecTask Commission. The main point here is that a large group of scientists who lack specific goals, responsibilities and roles are unlikely to successfully identify a need in the first place, and neither are they likely to achieve consensus or bring about recommendations that will be utilized by the Earth Science community.

\section{The current financial organization is peculiar}

At present, it appears that the TecTask Secretary is the only member of the TecTask EC that has the signing authority over the TecTask account (which is held by a German bank). However, the Chair and the Treasurer are accountable for the finances of the Commission and hence should be the EC members with signing authority over the account.

\section{The review of TecTask Annual Reports by the IUGS EC was unsatisfactory}

The EC procedures for review of the annual reports of its
Commissions should be reviewed and revised. Annual reports should be required to complete a budget template in order to ensure that IUGS funds are fully accounted for. As it stands, the annual TecTask reports did not provide basic financial information including the amount of IUGS funding received for the year; how that funding was spent; and the overall financial standing of the commission. The IUGS is financially liable for its commissions, and hence this information should be required on an annual basis. IUGS should not distribute new funds to commissions without knowing exactly how the funds from the previous year were utilized.

A template for annual reports would also facilitate a better oversight of commissions and ensure that basic information is reported on an annual basis. Examples of items that should be required in such a template would include a summary of goals for the year as stated in the previous report, and progress toward meeting these goals.

At present, there is no system in place to check for annual progress. Tectask Annual Reports were typically largely copies of the previous year's reports. Goals for the year remained largely unchanged despite there being no obvious progress made toward meeting most of those goals. But the ECs procedures for reviewing the annual reports meant that the lack of progress was never identified, and neither were concerns or questions referred back to the TecTask leadership.

A related problem is that the EC did not ensure that new and incoming officers of TecTask were made aware, ahead of their appointment, of the goals and rules governing IUGS Commissions. In addition, the EC failed to provide oversight or critical formal feedback to TecTask. As a result, over the years the leadership of TecTask came to an understanding of the goals of the Commission that was significantly at odds with the IUGS guidelines. Annual correspondence with the Commission including ensuring that incoming officers were aware of the goals of and rules governing IUGS Commissions, could have prevented this disconnect from developing.

\section{Conclusions and Recommendations}

The Ad-hoc Review Committee has recognized that a number of past and current TecTask activities - although relevant for the geological community - do not align with the overall goals of an IUGS commission. The Committee acknowledges the efforts made by the IUGS TecTask Commission leadership over the years to fill the void caused by the absence of an international scientific association covering the fields of structural geology and tectonics. We argue that the establishment of such an association should be a primary concern for the Earth Sciences community. Such a confusing situation cannot continue: the goals of TecTask should be aligned with the IUGS overall tasks and with the procedures and objectives of other successful IUGS bodies, i.e. the formal establishment of best-practice proce- 
dures, geological standards and baselines for the benefit of the global Earth sciences community.

It is recommended that:

1. TecTask defines two or (at most) three main goals

These goals should be presented to the IUGS EC at the $72^{\text {nd }}$ meeting of the Executive Committee of the IUGS in Potsdam in January, 2018 for discussion and ratification. The goals should be consistent with the mandate of the IUGS and with the aims of IUGS Commissions; they should have clearly defined time lines with the aim being to complete these tasks by the 2020 Delhi IGC; and a clear set of deliverables should by defined for each goal. The 2020 Delhi IGC will represent an opportunity to review the progress of TecTask and evaluate whether or not the commission should be continued.

2. The leadership of TecTask is reduced to a small, dedicated group

Such group must be aligned with and strongly committed to the successful completion of TecTask's new goals.

3. The process for the review of annual reports is revised
The ARC has concluded that the TecTask annual reports did not provide enough information and that there was not enough feedback from the IUGS Executive Committee to TecTask. The evaluation process of annual reports and the ensuing communication with commissions and other IUGS bodies need to be reviewed and improved.

The IUGS ARC members offer special thanks to the Department of Earth Sciences of the University of Turin for hosting the Review Committee.

October 4, 2017

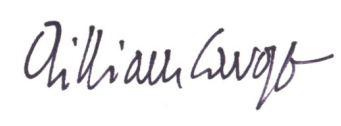

Prof. William Cavazza

ARC Chairperson

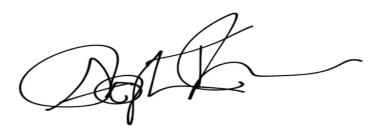

Prof. Stephen Johnston ARC Secretary 